\title{
An unexpected cause of severe and refractory PTH-independent hypercalcemia: case report and literature review
}

Dimitris Varvaki Rados', Tania Weber Furlanetto ${ }^{1,2}$

\begin{abstract}
SUMMARY
Hypercalcemia is a common condition in the internal medicine practice. Sometimes its cause is not readily apparent, so extensive investigation is appropriate. Here we report an unexpected cause for hypercalcemia in an elderly woman. The case of an 82-years old woman with PTH-independent hypercalcemia, lymphocytosis, normal serum 1,25 (OH)vitamin D levels, and low serum PTHrp levels, is described. Medical history and complementary investigation were unremarkable, except for increased metabolic activity in the glutei regions, as measured by whole body 18F-FDG PET-CT. Reviewing her medical history, her sister recalled that she had been submitted to intramuscular methylmethacrylate injections, for cosmetic purposes, five years before presentation, which was confirmed by muscle biopsy. Low calcium intake, parenteral bisphosphonates, calcitonin, and glucocorticoids were used to control serum calcium levels. Methylmethacrylate injections, used cosmetically, are a new cause for hypercalcemia, even after many years. Hypercalcemia was probably due to calcitriol overproduction in foreign body granulomas. Persistent reactive lymphocytosis could be a clue for this inflammatory cause of hypercalcemia. Arch Endocrinol Metab. 2015;59(3):277-80
\end{abstract}

1 Internal Medicine Division, Hospital de Clinicas de Porto Alegre (HCPA), Porto Alegre, RS, Brazil 2 Program in Medicine, Medical Sciences of Federal University of Rio Grande do Sul (UFRGS), Porto Alegre, RS, Brazil

Correspondence to:

Tania Weber Furlanetto

Serviço de Medicina Interna,

Hospital de Clínicas de Porto Alegre

Rua Ramiro Barcelos, 2350, sala 700 90035-903 - Porto Alegre, RS, Brazil tfurlanetto@hcpa.ufrgs.br

Received on Sept/24/2014 Accepted on Feb/20/2015

DOI: $10.1590 / 2359-399700000005$

\section{INTRODUCTION}

$\mathrm{H}$ ypercalcemia is a relatively common problem in clinical practice. Sometimes the cause is not readily apparent, so extensive investigation is appropriate. Here we report an unexpected cause for hypercalcemia in an elderly woman.

\section{CASE REPORT}

An 82-years old woman presented with recurrent episodes of confusion, delirium, and somnolence or stupor, for the past 6 months. Her previous medical history was unremarkable, except for previous 20 pack years smoking and systemic arterial hypertension. She has been using daily hydrochlorothiazide $25 \mathrm{mg}$, amiloride $2.5 \mathrm{mg}$, vitamin D3 $2000 \mathrm{IU}$, and calcium carbonate $2 \mathrm{~g}$. There were no previous surgeries or hospital admissions. The physical exam was unremarkable, except for low BMI $\left(17 \mathrm{Kg} / \mathrm{m}^{2}\right)$. Initial evaluation, as shown in table 1 , disclosed severe PTH-independent hypercalcemia, lymphocytosis, and no vitamin D intoxication. All her medications were stopped, and, as serum phosphate level was in the low reference range and tubular reabsorption of phosphate was low, a probable malignant cause was searched for. Computed tomography (CT) of neck, thorax, and abdomen, revealed a small swelling of the pyriform sinus, and simple renal and ovarian cysts, up to $1.2 \mathrm{~cm}$; fiberoptic indirect and direct laryngoscopy identified no lesion, and biopsies of the pyriform sinus region had no tumor; bone scintigraphy, bone marrow aspiration, peripheral blood cells immunophenotyping, serum and urine protein electrophoresis, and upper gastrointestinal endoscopic study were normal. Transvaginal pelvic ultrasound confirmed the presence of a simple ovarian cyst. A whole body 18F-FDG PET-CT showed extensive and symmetric increased metabolic activity in the glutei regions, as shown in figure 1A. At inspection, bilateral glutei regions were normal, but irregular 2-3 cm hard nodules were identified by palpation. The abdominal CT was reviewed with identification of extensive areas of increased density and loss of definition of both gluteus maximus, as shown in figure $1 \mathrm{~B}$. 
Table 1. Laboratory data at admission

\begin{tabular}{lcc}
\hline & Result & Reference range \\
\hline Hemoglobin (g/dL) & 11.1 & $11.0-16.0$ \\
Hematocrit (\%) & 33.2 & $35-47$ \\
White cells (per mL) & 9,640 & $3,600-11,000$ \\
Neutrophyls (per mL) & 4,400 & $1,500-7,000$ \\
Lymphocytes (per mL) & 4,300 & $1,000-2,900$ \\
Platelets (1,000/mL) & 188 & $150-400$ \\
Creatinine (mg/dL) & 1.32 & $0.6-1.1$ \\
Sodium (mmol/L) & 142 & $136-145$ \\
Potassium (mmol/L) & 3.4 & $3.5-5.1$ \\
Bicarbonate (mmol/L) & 27 & $22-30$ \\
Calcium (mg/dL) & 13.7 & $8.6-10.0$ \\
Phosphorus (mg/dL) & 2.5 & $2.4-5.1$ \\
Magnesium (mg/dL) & 1.8 & $1.2-2.7$ \\
Alkaline phosphatase (U/L) & 68 & $20-130$ \\
PTH (pg/mL) & 2.8 & $14-72$ \\
25-OH-vitamin D (ng/mL) & 15.2 & $>20$ \\
AST (U/L) & 31 & $10-34$ \\
ALT (U/L) & 22 & $10-49$ \\
Albumin (g/dL) & 3.5 & $3.2-4.8$ \\
TRP (\%) & 59 & $85-100$ \\
\hline
\end{tabular}

PTH: parathyroid hormone; AST: aspartate transaminase; ALT: alanine transaminase; TRP: tubular reabsorption of phosphate.

Reviewing the medical history, her sister recalled that she has been submitted to intramuscular methylmethacrylate injections in the glutei regions for cosmetic reasons, five years before presentation. A gluteus muscle biopsy showed chronic inflammation with fibrosis, histiocytes, lymphocytes, and giant cells around foreign body, compatible with methylmethacrylate (Figure 2). A surgical consultation was made, but resection of the lesions was not indicated due to its extension. At this point serum PTHrp and calcitriol levels were available: PTHrp: below $0.8 \mathrm{pmol} / \mathrm{L}$ (reference range < $1.3 \mathrm{pmol} / \mathrm{L}$; chemiluminescence); calcitriol: $34 \mathrm{pg} /$ $\mathrm{mL}$ (reference range $18-78 \mathrm{pg} / \mathrm{mL}$; radioimmunoassay). Dietary calcium was reduced to $350 \mathrm{mg} /$ day, and $40 \mathrm{mg}$ of oral prednisone was prescribed for 10 days and increased to $80 \mathrm{mg} /$ day thereafter. Two weeks later, serum calcium levels were within the reference range, as well as blood lymphocytes, so prednisone was tapered to $20 \mathrm{mg}$ /day. During the investigation and follow-up, saline infusion, calcitonin, and intravenous bisphosphonates were also used. These data are depicted in figure 3 . Twelve months later, the patient had normal serum calcium levels. Written informed consent was obtained to report this case.
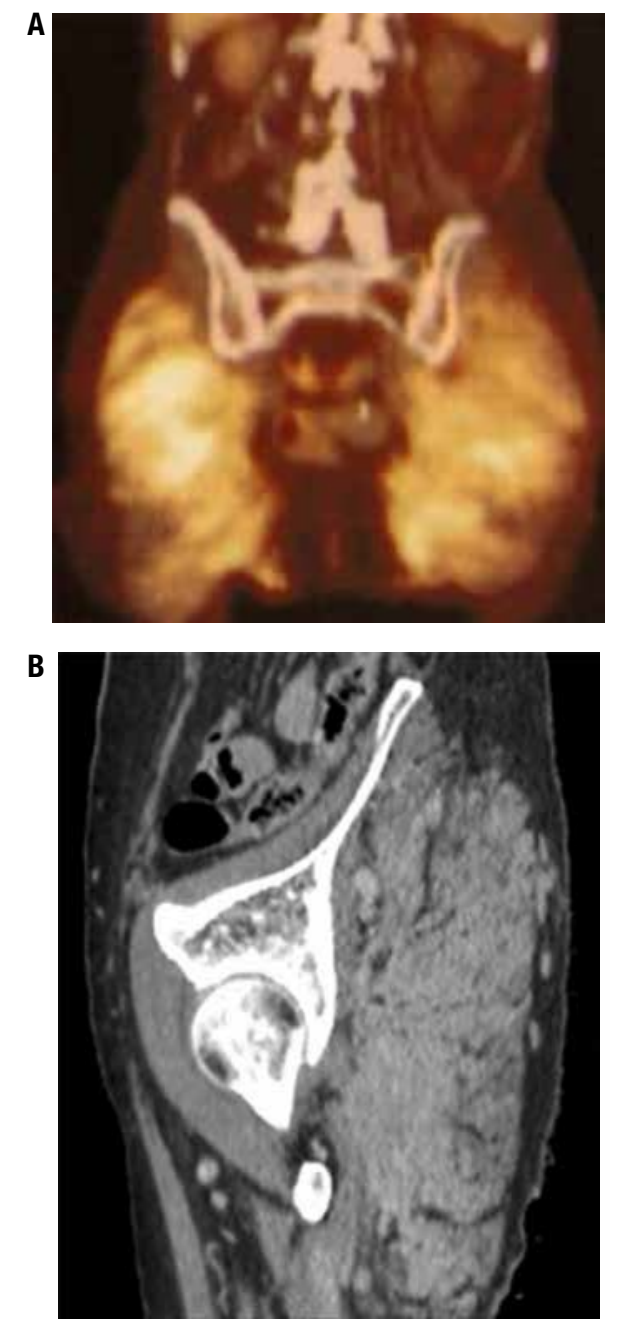

Figure 1. Abnormality of glutei regions, five years after the intramuscular injections of methylmethacrylate. (A) Increased metabolic activity, as shown by 18-FDG PET-CT Study. (B) Extensive areas of increased density and loss of definition of the gluteus maximus, as shown by computed tomography.

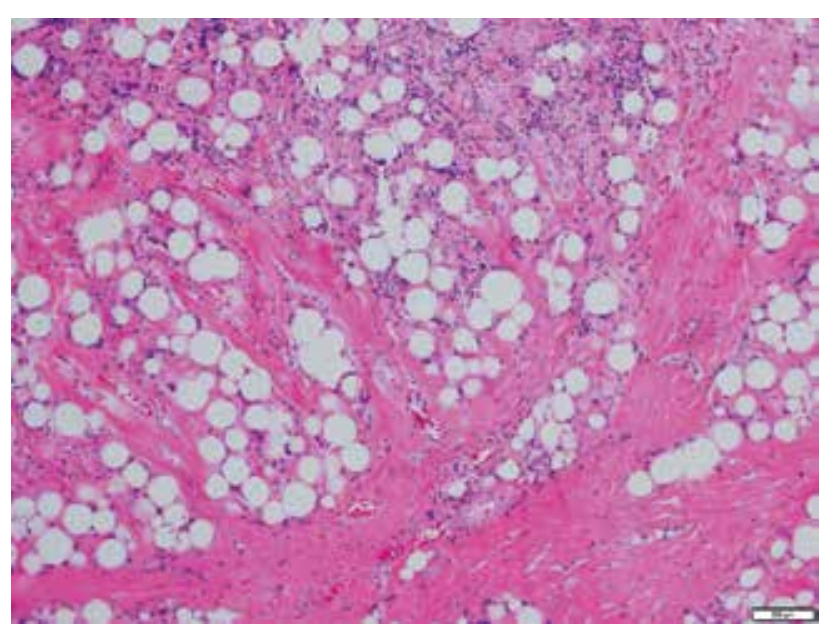

Figure 2. Fibrosis and foreign body granulomatous reaction in gluteus muscle, five years after the intramuscular injections of methylmethacrylate. Hematoxylin and eosin stain, 10x. 

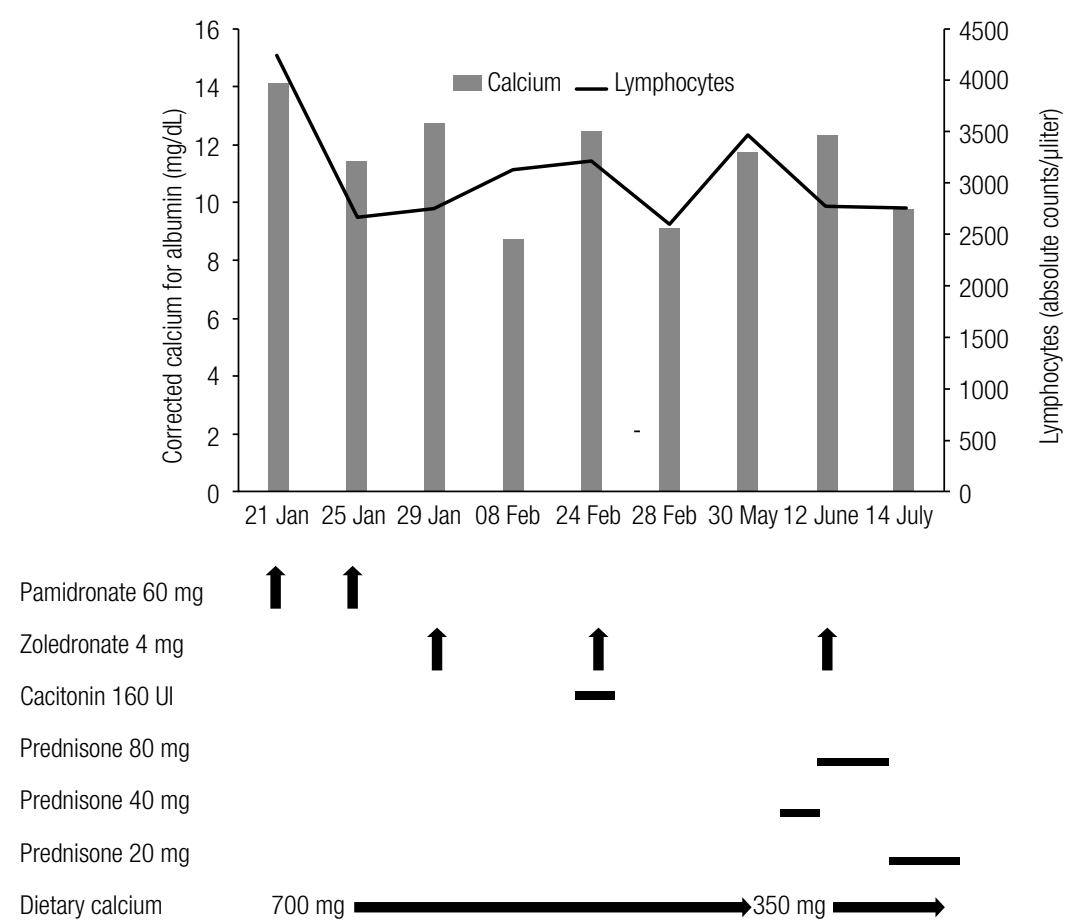

Figure 3. Serum calcium corrected for albumin, peripheral blood lymphocytes, and treatment used for hypercalcemia, five years after the intramuscular injections of methylmethacrylate for cosmetic reasons.

\section{DISCUSSION}

In this report, a patient with PTH-independent hypercalcemia, and non-monoclonal persistent lymphocytosis is described. The investigation was negative for cancer and identified a PTH-independent, calcitriol-mediated hypercalcemia secondary to foreign body granulomas induced by intramuscular methylmethacrylate, with a good response to oral prednisone. The milk-alkali syndrome was excluded due to the normal serum bicarbonate levels at presentation.

The most widely known granulomatous diseases causing hypercalcemia are tuberculosis, sarcoidosis, and systemic fungal infections. Hypercalcemia in these situations is mostly attributed to increase in serum calcitriol levels, due to higher lalfa-hydroxylase activity in activated macrophages $(1,2)$, although, in some patients, hypercalcemia has been associated with PTHrp $(3,4)$. In several patients with granulomatous diseases and hypercalcemia, serum calcitriol levels have been in the normal range, which are inappropriately high, considering the low serum PTH levels (2). In these patients, serum vitamin $\mathrm{D}$ levels probably did not reflect tissue-level vitamin D (5).
Less commonly, other causes of granulomatous reactions have been associated with hypercalcemia, also with elevated calcitriol levels (1), as foreign body reaction to silicone (6), paraffin (7), and talc (8). In our patient, hypercalcemia was very probably mediated by calcitriol, as serum PTH and PTHrp levels were not detectable (5).

Recently four cases of hypercalcemia associated with methylmethacrylate injection have been described in Buenos Aires, Argentina (9). All patients had severe hypercalcemia, low or suppressed PTH levels, and high or inappropriately normal serum calcitriol levels (9).

Some features of the present case are not completely clear. Although at presentation the patient was taking 2000 IU of vitamin D3 daily, serum 25-OH-vitamin D levels were low. Several factors might have contributed to it: irregular intake of the prescribed vitamin $\mathrm{D}$, decreased vitamin $\mathrm{D}$ absorption, and increased conversion of 25-OH-vitamin D to its metabolites in the granulomas. Also, serum phosphate levels and tubular reabsorption of phosphate were lower than expected. Bisphosphonate use and malnutrition could explain the serum phosphate levels, but not the increased phosphate excretion. 
At last, our patient had high normal blood lymphocytes count, after elevated doses of glucocorticoids, which are usually associated with low lymphocyte counts (10). As shown in figure 3 , lymphocytes counts and serum calcium seemed to change together, which could be a clue to the underlying chronic inflammatory condition, as the cause for hypercalcemia.

In conclusion, we report a case of PTH-independent hypercalcemia caused by foreign body granulomas, five years after the cosmetic intra-muscular methylmethacrylate injection in the glutei regions, with poor response to bisphosphonates, and good response to glucocorticoids and low calcium intake.

Funding source: National Council for Research and Development, CNPq, Brazil.

Disclosure: no potential conflict of interest relevant to this article was reported.

\section{REFERENCES}

1. JacobsTP, Bilezikian JP. Clinical review: rare causes of hypercalcemia. J Clin Endocrinol Metab. 2005;90(11):6316-22.
2. Shrayyef MZ, DePapp Z, Cave WT, Wittlin SD. Hypercalcemia in two patients with sarcoidosis and Mycobacterium avium intracellulare not mediated by elevated vitamin D metabolites. Am J Med Sci. 2011;342(4):336-40.

3. Fierer J, Burton DW, Haghighi P, Deftos LJ. Hypercalcemia in disseminated coccidioidomycosis: expression of parathyroid hormone-related peptide is characteristic of granulomatous inflammation. Clin Infect Dis. 2012;55(7):e61-6.

4. Krikorian A, Shah S, Wasman J. Parathyroid hormone-related protein: an unusual mechanism for hypercalcemia in sarcoidosis. Endocr Pract. 2011;17(4):e84-6.

5. Berlin JL, Shantha GP,Yeager H, Thomas-Hemak L. Serum vitamin D levels may not reflect tissue-level vitamin $D$ in sarcoidosis. BMJ Case Rep. 2014;2014. pii: bcr2014203759.

6. Schanz J, Flux K, Kircher C, Tsioga M, Hartschuh W, Nawroth PP, et al. Mirror, mirror on the wall: hypercalcemia as a consequence of modern cosmetic treatment with liquid silicone. Med Sci Monit. 2012;18(2):CS5-7.

7. Gyldenløve M, Rørvig S, Skov L, Hansen D. Severe hypercalcaemia, nephrocalcinosis, and multiple paraffinomas caused by paraffin oil injections in a young bodybuilder. Lancet. 2014;383(9934):2098.

8. Woywodt A, Schneider W, Goebel U, Luft FC. Hypercalcemia due to talc granulomatosis. Chest. 2000;117(4):1195-6.

9. Negri AL, Rosa Diez G, Del Valle E, Piulats E, Greloni G, Quevedo $A$, et al. Hypercalcemia secondary to granulomatous disease caused by the injection of methacrylate: a case series. Clin Cases Miner Bone Metab. 2014;11(1):44-8.

10. Ashwell JD, Lu FW, Vacchio MS. Glucocorticoids in T cell development and function*. Annu Rev Immunol. 2000;18:309-45. 